\title{
ACTIVE FAULT DIAGNOSIS BASED ON STOCHASTIC TESTS
}

\author{
NIELS K. POULSEN*, HENRIK NIEMANN ** \\ * Department of Informatics and Mathematical Modeling \\ Technical University of Denmark, Building 321, DK-2800 Kgs. Lyngby, Denmark \\ e-mail: nkp@imm.dtu.dk \\ ${ }^{* *}$ Department of Electrical Engineering, Automation and Control \\ Technical University of Denmark, Building 326, DK-2800 Kgs. Lyngby, Denmark \\ e-mail: hhneelektro.dtu.dk
}

\begin{abstract}
The focus of this paper is on stochastic change detection applied in connection with active fault diagnosis (AFD). An auxiliary input signal is applied in AFD. This signal injection in the system will in general allow us to obtain a fast change detection/isolation by considering the output or an error output from the system. The classical cumulative sum (CUSUM) test will be modified with respect to the AFD approach applied. The CUSUM method will be altered such that it will be able to detect a change in the signature from the auxiliary input signal in an (error) output signal. It will be shown how it is possible to apply both the gain and the phase change of the output signal in CUSUM tests. The method is demonstrated using an example.
\end{abstract}

Keywords: active fault diagnosis, parametric faults, stochastic change detection, closed-loop systems, parameterization.

\section{Introduction}

The area of active fault diagnosis (AFD) has been considered in a number of papers (Campbell, Horton and Nikoukhah, 2002; Campbell, Horton, Nikoukhah and Delebecque, 2000; Campbell and Nikoukhah, 2004b; Kerestecioglu and Zarrop, 1994; Niemann, 2006; Nikoukhah, 1994; Nikoukhah, 1998; Nikoukhah, Campbell and Delebecque, 2000) and books (Campbell and Nikoukhah, 2004a; Kerestecioglu, 1993; Zhang, 1989).

AFD is based on the inclusion of an auxiliary (test) input signal into the system. The auxiliary input can be injected in either the open-loop system or the closed-loop system. As the output from the diagnosis system, a standard residual signal known from the passive FDI approach is applied (Frank and Ding, 1994). Using the AFD approach from (Niemann, 2005; Niemann, 2006), the auxiliary input is injected into the closed-loop system in such a way that the residual is decoupled from the auxiliary input in the nominal case. In the case of a parameter change, the residual will contain a component related to the auxiliary input. It turns out that this approach connects AFD with dual YJBK parameterization (after Youla, Jabr, Bongiorno and Kucera), (Niemann, 2003; Tay, Mareels and Moore, 1997). The transfer function from the auxiliary input to the residual is equivalent to dual YJBK transfer function in the dual YJBK parameterization, i.e., a parameterization of all systems stabilized by a given feedback controller. Here, in connection with AFD, this transfer function will be named the fault signature matrix (Niemann, 2005; Niemann, 2006). Change/fault detection, as well as change/fault isolation, is based directly on the fault signature matrix.

There are two main approaches to AFD. In one approach, which was originally derived by Zhang (Zhang, 1989), the auxiliary input is designed with respect to fast fault diagnosis/multi model selection. This is obtained by means of a dedicated design of the auxiliary input signal. This method was later investigated extensively in (Campbell and Nikoukhah, 2004a; Kerestecioglu, 1993; Kerestecioglu and Zarrop, 1994),

In the other AFD approach, that in (Niemann, 2005; Niemann, 2006), a periodic auxiliary input is applied. This approach was also applied in (Niemann and Poulsen, 2005). In the normal situation there is no trace of the auxiliary input in the residual. A change in the system, e.g., due to parametric changes/faults, will result in a change in the signature in such a way that the residual will contain a component of the periodic input signal. 
Using the AFD approach from (Niemann, 2005; Niemann, 2006), the auxiliary input is decoupled in the output/residual in the nominal case. The detection of parameter changes can then be done by detecting a signature from the auxiliary input in the residual signal. Another approach is to use a filter/observer to estimate the periodic signature with the known frequency directly. This approach will not be considered in this paper. Instead, the classical CUSUM method (Basseville and Nikiforov, 1993; Gustafsson, 2000) will be applied for change detection. The CUSUM method will be modified to detect changes based on the periodic auxiliary input. This modification can be done in different ways. It is possible to let the CUSUM test be based only on the amplitude/gain of the signature in the residual signal from the auxiliary input, or it can be based on both the gain and the phase shift in the signature signal. Using both the gain and the phase shift for change detection, it will also be possible to isolate changes in different parameters. From a theoretical point of view, it will be possible to isolate an unlimited number of parameter changes. In practice, however, there will be an upper bound on the number of parameters that can be isolated based on a single periodic auxiliary input. This number will depend strongly on the signal/noise ratio.

Only the SISO case will be considered in this paper, but it is possible to extend the results to the MIMO case without any major difficulties. Further, only periodic stationary auxiliary inputs will be applied as considered in (Kerestecioglu and Cetin, 2004) in connection with AFD. In (Kerestecioglu and Cetin, 2004), it was shown that the optimal stationary auxiliary inputs are linear combinations of a limited number of periodic signals. In some cases, the optimal auxiliary input consists of only a single periodic signal. In this paper we will only consider auxiliary inputs based on a single periodic signal. However, it is possible to extend the results derived in this paper to the case where more than a single periodic input are applied. Some preliminary results were given in (Poulsen and Niemann, 2007).

The outline of this paper is as follows: In Section 2 the system set-up is given followed by a short description of the AFD set-up applied in Section 3 . Statistical test methods applied in connection with AFD are considered in Section 4 In Section 5, an evaluation of the derived fault detectors is given. The developed methods are applied using a simple example in Section 6 The paper is completed with a conclusion in Section 7

\section{System set-up}

Let a general system be given by

$$
\Sigma_{P, \theta}:\left\{\begin{array}{l}
e_{t}=G_{e d}(\theta) d_{t}+G_{e u}(\theta) u_{t}, \\
y_{t}=G_{y d}(\theta) d_{t}+G_{y u}(\theta) u_{t},
\end{array}\right.
$$

where $d_{t} \in \mathbb{R}^{r}$ is a disturbance signal vector, $u_{t} \in \mathbb{R}$ is the control input signal, $e_{t} \in \mathbb{R}^{q}$ is the external output signal vector to be controlled and $y_{t} \in \mathbb{R}$ is the measurement signal. The system description in (1) may depend on a number $(k)$ of parameters. Let $\theta_{i}, i=1, \ldots, k$ denote the deviations away from the nominal values, i.e., $\theta_{i}=0, i=1, \ldots, k$ in the nominal case. For notational convenience, arrange the deviations in a vector:

$$
\theta=\left(\theta_{1}, \ldots, \theta_{i}, \ldots, \theta_{k}\right)^{T} .
$$

Furthermore, let

$$
\vartheta_{i}=\left(0, \ldots, \theta_{i}, \ldots, 0\right)^{T},
$$

which represents the situation with a change in precisely one parameter. In many cases it will be possible to give an explicit expression of the connection between the system and the parametric change as described in (Niemann, 2006; Niemann and Poulsen, 2005). Such an explicit description is not needed in this paper.

Let the system be controlled by a stabilizing feedback controller

$$
\Sigma_{C}:\left\{u_{t}=K y_{t}\right.
$$

The results derived in this paper are based on the system set-up given above for discrete-time systems. However, the results are easily adapted to continuous-time systems.

2.1. Coprime factorization. Let $G_{y u}(0)$ be the nominal system from (1) and let $K$ be a stabilizing controller from (2). Assume that a coprime factorization of $G_{y u}(0)$ and $K$ exists and

$$
\begin{aligned}
G_{y u}(0) & =\frac{N}{M}, \quad N, M \in \mathcal{R} \mathcal{H}_{\infty}, \\
K & =\frac{U}{V}, \quad U, V \in \mathcal{R} \mathcal{H}_{\infty},
\end{aligned}
$$

where the four transfer functions $(N, M, U$ and $V)$ in (3) must satisfy the Bezout equation

$$
1=M V-N U
$$

see (Tay et al., 1997).

\section{AFD set-up}

Now, consider the AFD set-up described in (Niemann, 2006; Niemann and Poulsen, 2005). The set-up is shown in Fig. 1. The diagram includes the residual $\varepsilon_{t}$ and an auxiliary input $\eta_{t}$.

The residual $\varepsilon_{t}$ for $\Sigma_{P, \theta}$ in (1) is given by

$$
\varepsilon_{t}=M y_{t}-N u_{t} .
$$




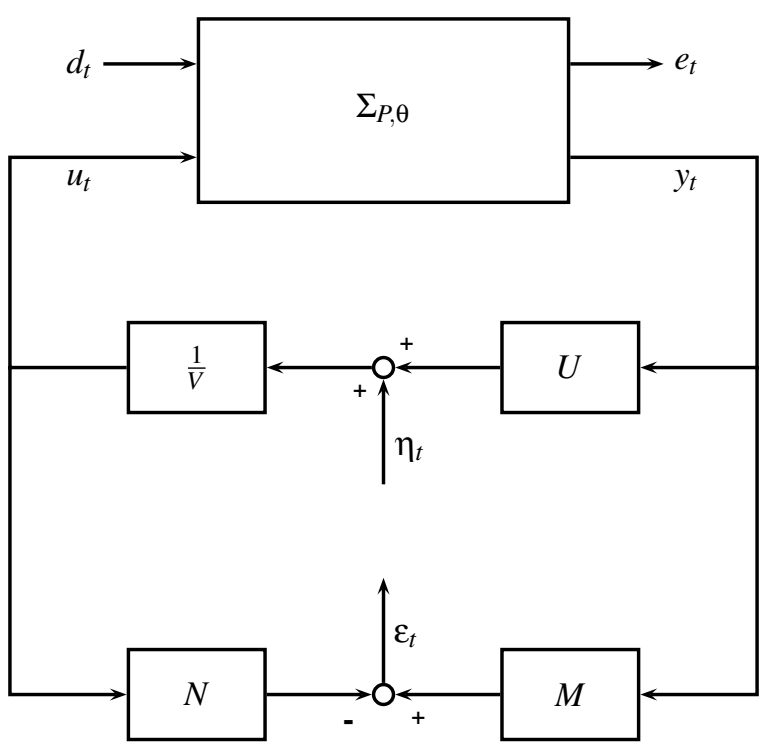

Fig. 1. Block diagram for an AFD set-up based on a closed-loop system. The set-up includes a residual $\varepsilon_{t}$ and an external input signal $\eta_{t}$

Notice that it is the same residual generator as that used in connection with passive fault diagnosis (Frank and Ding, 1994). A more detailed discussion of the AFD set-up applied is given in (Niemann, 2006; Niemann and Poulsen, 2005).

Based on the feedback system in Fig. 1, the transfer functions from the two inputs $d_{t}, \eta_{t}$ to the two outputs $e_{t}, \varepsilon_{t}$ are given by (Niemann, 2005; Niemann and Poulsen, 2005)

$$
\Sigma_{\mathrm{FD}}:\left\{\begin{array}{l}
e_{t}=P_{e d}(\theta) d_{t}+P_{e \eta}(\theta) \eta_{t}, \\
\varepsilon_{t}=P_{\varepsilon d}(\theta) d_{t}+P_{\varepsilon \eta}(\theta) \eta_{t},
\end{array}\right.
$$

where

$$
\begin{aligned}
& P_{e d}=G_{e d}(\theta)+\frac{G_{e u}(\theta) U G_{y d}(\theta)}{V-G_{y u}(\theta) U}, \\
& P_{e \eta}=\frac{G_{e u}(\theta)}{V-G_{y u}(\theta) U}, \\
& P_{\varepsilon d}=\frac{G_{y d}(\theta)}{V-G_{y u}(\theta) U}, \\
& P_{\varepsilon \eta}=-\frac{N-G_{y u}(\theta) M}{V-G_{y u}(\theta) U} .
\end{aligned}
$$

The system $\Sigma_{\mathrm{FD}}$ is shown in Fig. 2, The transfer function from the input signal $\eta_{t}$ to the residual $\varepsilon_{t}$ is equal to the dual YJBK transfer function (Niemann, 2005; Niemann and Poulsen, 2005). An important thing in this con-

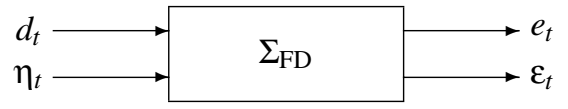

Fig. 2. System set-up for active fault diagnosis.

nection is that the dual YJBK transfer function is equal to zero in the nominal case. This means that the transfer function from the auxiliary input $\eta_{t}$ to the residual $\varepsilon_{t}$ will be zero in the nominal case.

In (Niemann, 2005; Niemann and Poulsen, 2005), the dual YJBK transfer function was called the fault signature matrix in connection with AFD. Here it is a transfer function. In the following, it will be denoted by $S(\theta)$, where $S(\theta)=P_{\varepsilon \eta}(\theta)$. An explicit equation for $S(\theta)$ was derived in (Niemann, 2003; Niemann, 2006).

The fault signature matrix is a measure of the effect of parameter variation on the closed-loop stability. Large $S$ indicates that parameter variations have a major influence on the system.

\section{Change detection}

The implementation of the AFD set-up is not unique, because the coprime factorization is not unique. This will give an extra freedom in the implementation of the AFD set-up. For example, it is possible to base the coprime factorization of $G_{y u}$ on a Kalman filter. In the nominal case this will produce a residual signal with well-defined properties (such as being a white noise sequence). If the residual signal in the normal situation is not white, then a filter which extracts the white innovation from the residual can be applied. The design freedom introduced in connection with the coprime factorization of the system and controller will not be discussed further in this paper. It will be assumed that the residual signal is the innovation signal from a Kalman filter.

In a passive scheme the detection is often based on a change in the statistics (the mean, variance, correlation or spectral properties) of the residual sequence. In an active scheme an auxiliary signal is introduced and the residual in (6) takes then the following form:

$$
\varepsilon_{t}=S(\theta) \eta_{t}+\xi_{t},
$$

where $\xi_{t} \in N\left(0, \sigma_{0}^{2}\right)$ and it is white in the nominal case. It is clear that a detection can be based on the change in the statistics of $\xi_{t}$. Here in this paper we will follow another approach. From (7) we have that $S(\theta)$ is zero in the nominal case, i.e.,

$$
S(0)=0 .
$$

Further, $S(\theta)$ reflects the importance (of the change) in the control. It is clear from this observation that $S(\theta)$ is very important in connection with active change detection (or 
active fault detection). A direct consequence of (9) is the following condition:

$$
\begin{aligned}
& S(\theta)=0 \text { for } \theta=0, \\
& S(\theta) \neq 0 \text { for } \theta \neq 0 .
\end{aligned}
$$

The detection (isolation) of parameter variations can then be based on the following null and alternative hypotheses:

$$
\begin{array}{ll}
H_{0}: & S(\theta)=0, \\
H_{1}: & S(\theta) \neq 0 .
\end{array}
$$

Tests of the above null hypothesis and its alternative can be done by on-line evaluation of the residual signal with respect to the signature from the auxiliary input in the residual $\varepsilon_{t}$. Consequently, the auxiliary signal is chosen in such way that the signature in the residual is distinctive. This is in contrast to methods in which the objective is a change in the statistics (normally, the mean and variance) of the residual. For this reason (and others, explained later) the auxiliary input is chosen as being a periodic signal given by

$$
\eta_{t}=a_{\omega} \sin \left(\omega_{0} t\right)
$$

where the amplitude $a_{\omega}$ and the frequency $\omega_{0}$ are the tuning parameters in the auxiliary input. The specific signature in the residual of this signal is particularly easy. Using the auxiliary input given by (12), the residual signal is given by

$$
\varepsilon_{t}=\xi_{t}, \quad \xi_{t} \in N\left(0, \sigma_{0}^{2}\right)
$$

in the nominal case. If the parameter has changed (from nominal values), we have

$$
\varepsilon_{t}=a_{\omega}|S| \sin \left(\omega_{0} t+\phi\right)+\xi_{t}, \quad \xi_{t} \in N\left(m, \sigma_{1}^{2}\right),
$$

where $|S|$ and $\phi$ are respectively the (non-zero) gain and phase shift through the fault signature matrix $S$ at the chosen frequency $\omega_{0}$. For brevity, we have omitted the dependency of $\theta$ and $\omega_{0}$ in $S=S\left(\theta, \omega_{0}\right), \phi=\phi\left(\theta, \omega_{0}\right), m=m(\theta)$ and $\sigma_{1}=\sigma_{1}(\theta)$. In general, $m$ will be zero. Both the amplitude and phase of the periodic signal in $\varepsilon_{t}$ depend on $\theta$ and on the chosen frequency, $\omega_{0}$. The periodic signal in $\varepsilon_{t}$ is the signature of the periodic auxiliary input $\eta_{t}$.

The detection of parameter changes is then based on the detection of the signature from $\eta_{t}$ in $\varepsilon_{t}$. Further, the isolation of parameter changes may be possible from an investigation of the amplitude and phase of the signature in $\varepsilon_{t}$. In some cases it may be necessary to include more than one single periodic signal in $\eta_{t}$ in order to isolate different parameter changes. Here we will only consider a single periodic auxiliary input signal.

The most direct way to detect a signature in $\varepsilon_{t}$ is by a visual inspection of $\varepsilon_{t}$. However, in general this will not be possible due to the noise component $\xi_{t}$ in $\varepsilon_{t}$. Further, the amplitude of $\eta_{t}$ is selected as small as possible to avoid to "disturb" the output $e_{t}$ too much. This will give a signature in $\varepsilon_{t}$ with a small amplitude that is not directly visible in general. Instead, other methods need to be applied.

The selection of the amplitude and the frequency of the periodic input signal $\eta_{t}$ is not trivial. The selection of $a_{\omega}$ and $\omega_{0}$ needs to be done with respect to a number of conditions. The choice of the amplitude is given by the tolerated increase in power in $e_{t}$ due to the auxiliary signal in the normal case. It is clear that a higher amplitude will increase the speed of detection and enable the algorithm to detect small parameter changes. The selection of $\omega_{0}$ has to be done with respect to the following conditions:

1. Maximize the signal-to-noise ratio between the signal and the noise components in the residual $\varepsilon_{t}$ if a parameter has changed. The signal component is the signature from the auxiliary signal $\eta_{t}$, and the noise component is the effect from the disturbance input $d_{t}$.

2. Minimize the effect from $\eta_{t}$ on the external output $e_{t}$ in the normal situation.

3. The selection of the frequency must be done in such a way that it will be possible to discriminate between different types of changes in the signature (the transfer function from $\eta_{t}$ to $\varepsilon_{t}$ ).

By using the closed-loop transfer function from the AFD set-up, the above conditions can be formulated as follows:

Condition 1 is equivalent to

$$
\max _{\omega} \frac{\left|S(\theta, \omega) \eta_{t}\right|}{\left|P_{\varepsilon d}(\theta, \omega) d_{t}\right|}
$$

Condition 2 is equivalent to

$$
\min _{\omega}\left|P_{e \eta}(\theta, \omega) \eta_{t}\right|
$$

Condition 3 specifies that the signature from $\eta_{t}$ in $\varepsilon_{t}$ given by (14) must be different for different parameter changes. This is satisfied if the amplitude and the phase change of the periodic signature in $\varepsilon_{t}$ are unique for a change in a single parameter.

Conditions 1 and 2 are related to change detection, whereas Condition 3 is only related to change isolation. A frequency $\omega_{0}$ that optimizes the first two conditions might not be optimal with respect to change isolation. It will therefore be obvious to change the frequency in the auxiliary input signal when a parameter change has been detected but not isolated.

4.1. Parameter change detection. Assume that the auxiliary input signal has been selected, i.e., the amplitude $a_{\omega}$ and the frequency $\omega_{0}$ in (12) have been specified. In this section we will focus on how the null and alternative hypotheses in (11) can be implemented. As mentioned in 
the previous section, the approach taken in this paper is to test whether the signature of the auxiliary input signal is present in the residual. To this end, the following two signals are formed:

$$
s_{t}=\varepsilon_{t} \sin \left(\omega_{0} t\right), \quad c_{t}=\varepsilon_{t} \cos \left(\omega_{0} t\right),
$$

where, according to (14) and some trigonometric relations,

$$
\begin{aligned}
& s_{t}=|S| \frac{a_{\omega}}{2}\left(\cos (\phi)-\cos \left(2 \omega_{0} t+\phi\right)\right)+\xi_{t} \sin \left(\omega_{0} t\right), \\
& c_{t}=|S| \frac{a_{\omega}}{2}\left(\sin (\phi)+\sin \left(2 \omega_{0} t+\phi\right)\right)+\xi_{t} \cos \left(\omega_{0} t\right) .
\end{aligned}
$$

From this it is clear that in the normal (or the fault-free) situation

$$
\begin{aligned}
& s_{t}=\xi_{t} \sin \left(\omega_{0} t\right) \in N\left(0, \sigma_{0}^{2} \sin ^{2}\left(\omega_{0} t\right)\right), \\
& c_{t}=\xi_{t} \cos \left(\omega_{0} t\right) \in N\left(0, \sigma_{0}^{2} \cos ^{2}\left(\omega_{0} t\right)\right) .
\end{aligned}
$$

Additionally, the two signals are white when a filter parameterization is applied. The time average variance is equal to $\frac{1}{2} \sigma_{0}^{2}$.

If a change has occurred then the fault signature matrix, $S$, is different from zero and the two detection signals, $s_{t}, c_{t}$, will have a constant, deterministic component:

$$
m\left(S(\theta), a_{\omega}\right)=\left[\begin{array}{c}
m_{s} \\
m_{c}
\end{array}\right]=|S| \frac{a_{\omega}}{2}\left[\begin{array}{c}
\cos (\phi) \\
\sin (\phi)
\end{array}\right] .
$$

This component can be used for detection and isolation.

Besides the mentioned component, the detector signals will also have a time varying deterministic component:

$$
|S| \frac{a_{\omega}}{2}\left[\begin{array}{c}
-\cos \left(2 \omega_{0} t+\phi\right) \\
\sin \left(2 \omega_{0} t+\phi\right)
\end{array}\right]
$$

which, on the (time) average, is zero. The effect of this component can be eliminated by means of an average or integration technique such as that in the CUSUM methodology.

In the literature the CUSUM technique is normally connected to the detection of a change in the mean and/or variance in a signal. In the normal situation it is assumed that the signal is white and has a specific mean or variance (see (Basseville and Nikiforov, 1993) or (Gustafsson, 2000)). The detection is an implementation of a sequentiel test in which the inspection data are successively increased. CUSUM methods are normally based on simple (specified) null hypotheses and simple (specified) alternatives which have to be given as tuning parameters. A simple alternative then forms a situation that should be detected. In a heuristic setting CUSUM methods can be regarded as being a test of whether the slope of the integral of the signal in question exceeds a certain critical value. In this work we have transformed the problem and are testing whether the mean of the vector

$$
\left[\begin{array}{l}
s_{t} \\
c_{t}
\end{array}\right]
$$

has a zero mean (vector) or has the component given in (19).

Introduce the tuning parameters $\beta$ and $\gamma$. The detection can be implemented as a CUSUM detection given by

$$
z_{t+1}=\max \left(0, z_{t}+\left[\frac{\delta_{t}}{\sigma_{1}}-\frac{1}{2} \gamma\right]\right)
$$

where

$$
\delta_{t}=\left[\begin{array}{c}
s_{t} \\
c_{t} \\
-s_{t} \\
-c_{t}
\end{array}\right], \quad \sigma_{1}^{2}=\frac{1}{2} \sigma_{0}^{2} .
$$

The hypothesis $H_{0}$ is accepted if $z_{t}$ is smaller than the threshold $h$, i.e.,

$$
z_{t} \leq \frac{\log (B)}{\gamma}=h
$$

where the inequality is to be understood elementwise. The parameter $B$ in this CUSUM detector is related to the average length between false detections. The other parameter, $\gamma$, is chosen as a typical lower limit of changes to be detected. Furthermore, note that the time average variance of $c_{t}$ and $s_{t}$ was used in (21). The time distance from the last zero crossing of the elements in $z_{t}$ forms an estimate of the time of change, $T_{d}$.

4.2. Parameter change isolation. The phase information can be utilized in the process of isolating the type of parameter changes. As illustrated in Fig. 3 (for a twoparameter problem), for each type of parameter change (and for fixed $\left.\omega_{0}\right)$ the fault signature matrix $S\left(\omega_{0}, \theta\right)$ forms a curve in the complex plane which passes through the origin for $\theta=0$. For brevity, we call these fault signature curves. The parameter change isolation can then be performed by estimating the fault signature matrix $S\left(\omega_{0}, \theta\right)$ and match it with the possible values. However, due to stochastic disturbances an estimate of $S$ will inherently be uncertain. Instead the estimate should be matched with the nearest fault signature curve, e.g., in a least squares sense. These curves will then divide the complex plane into double conic areas, each related to each type of parameter change. The isolation procedure will then be a classification determining the areas an estimate of $S$ belongs to.

In order to isolate changes in different parameters, they must have different effects on $S(\theta)$. If this is the case, in theory there are no limits on the number of parameter changes (occurring separately) that can be isolated, when both amplitude and phase information is applied. However, in practice only a limited number of parameter 


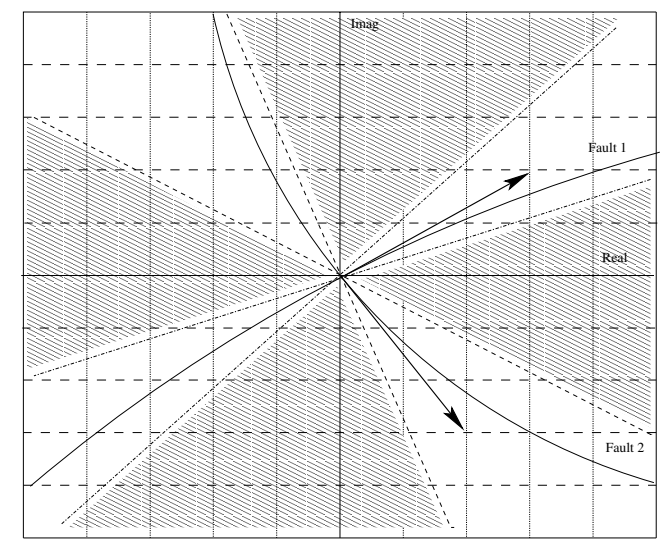

Fig. 3. For each type of errors (and for fixed $\omega_{0}$ ), the fault signature matrix, $S\left(\omega_{0}, \theta\right)$, forms a curve (shown as dashed lines) in the complex plane. The individual type of parameter changes forms a double-coned area (shown as shaded for Type 2 of parameter change).

changes can be isolated. This number will depend on the signal-to-noise ratio and on to what extent $S(\theta)$ is nonlinear. If the parameter change cannot be isolated at one frequency, then extra harmonics at different frequencies can be included in the auxilary signal.

For small parameter changes this classification can easily be automated by assigning a (unit) vector, $v_{i}, \quad i \in$ $\{1, \ldots, k\}$, to each type of parameter changes. The vectors are parallel to the tangent of $S\left(\omega_{0}, \vartheta_{i}\right)$ at the origin. In a more formal way, we define

$$
\tilde{v}_{i}=\left.\frac{\partial S\left(\vartheta_{i}\right)}{\partial \vartheta_{i}}\right|_{\vartheta_{i}=0}, \quad v_{i}=\frac{\tilde{v}_{i}}{\left\|\tilde{v}_{i}\right\|}
$$

Note that these vectors are parallel to the mean of the vector $\left(c_{t}, s_{t}\right)$ for the corresponding parameter change. Let $\hat{T}_{d}$ denote the estimate of the instant of the change. The vector

$$
v=\frac{1}{t-\hat{T}_{d}} \sum_{\tau=\hat{T}_{d}}^{t}\left[\begin{array}{l}
s_{\tau} \\
c_{\tau}
\end{array}\right] T_{s},
$$

which is the sum of the signals in (17) from the (estimated) instant of change $\hat{T}_{d}$ to the current time $t$, can be used to isolate the parameter change. In the deterministic and nominal cases $v$ will be a zero vector according to (17) and (20). The classification then reduces to finding the maximal projection among the types of parameter changes considered, i.e.,

$$
\hat{i}=\arg \max _{i \in\{1, \ldots, k\}} v^{T} v_{i} .
$$

\section{Evaluation of fault detectors}

It is relevant to evaluate fault detectors based on AFD by using a number of standard performance measures. Some of these performance measures are: the mean time between false alarms (MTFA) (or a similar false alarm rate (FAR)) and the mean time to detection (MTD). These performance measures can be determined from the average run length (ARL) function, which in general cannot be computed exactly. Instead, approximations of the performance measures can be derived, see, e.g., (Basseville and Nikiforov, 1993; Gustafsson, 2000).

Let $\mu_{\alpha}$ and $\sigma_{\alpha}^{2}$ be respectively the mean and the variance of each of the components in the increment

$$
\alpha_{t}=\frac{\delta_{t}}{\sigma_{1}}-\frac{1}{2} \gamma
$$

in the CUSUM test. An approximative solution to the ARL function is given by (Basseville and Nikiforov, 1993; Gustafsson, 2000)

$$
\begin{aligned}
& \hat{L}\left(\mu_{\alpha}, \sigma_{\alpha}, h\right) \\
& =\frac{\exp \left(-2\left(\frac{\mu_{\alpha} h}{\sigma_{\alpha}^{2}}+\frac{\mu_{\alpha}}{\sigma_{\alpha}} \beta\right)\right)-1+2\left(\frac{\mu_{\alpha} h}{\sigma_{\alpha}^{2}}+\frac{\mu_{\alpha}}{\sigma_{\alpha}} \beta\right)}{\frac{2 \mu_{\alpha}^{2}}{\sigma_{\alpha}^{2}}},
\end{aligned}
$$

where $h=\log (B) / \gamma$ is the detection threshold and $\beta=$ 1.166. This approximation is based on $\alpha_{t}$ being white, which is satisfied in a normal situation (and when a filter parameterization is applied). When a parameter has changed, this is only satisfied approximately.

Let $\alpha_{t}^{j}, j=1, \ldots, 4$ denote the components of the CUSUM increments. In the nominal situation we have

$$
\alpha_{t}^{j} \in \mathcal{N}\left(-\frac{\gamma}{2}, 1\right)
$$

and the mean time between false alarms, $\hat{\tau}_{\text {MTFA }}$, can the be estimated through

$$
\hat{\tau}_{\mathrm{MTFA}}=\hat{L}\left(-\frac{\gamma}{2}, 1, h\right) .
$$

When a parameter has changed, we have

$$
\alpha_{t}^{j} \in \mathcal{N}\left(\frac{|S(\theta)| a_{\omega} l^{j}}{2 \sigma_{1}}-\frac{1}{2} \gamma, \frac{\sigma_{f}^{2}}{\sigma_{0}^{2}}\right) .
$$

Here

$$
l=\left[\begin{array}{c}
\cos (\phi(\theta)) \\
\sin (\phi((\theta)) \\
-\cos (\phi(\theta)) \\
-\sin (\phi((\theta))
\end{array}\right]
$$

where $l^{j}$ is the $j^{\prime}$-th component of $l$. The mean time for 
detection, $\hat{\tau}_{\mathrm{MTD}}$, can be estimated from

$$
\hat{\tau}_{\mathrm{MTD}}=\min _{j} \hat{L}\left(\frac{|S(\theta)| a_{\omega} l^{j}}{2 \sigma_{1}}-\frac{\gamma}{2}, \frac{\sigma_{f}^{2}}{\sigma_{0}^{2}}, h\right) .
$$

An important thing with the AFD set-up used in this paper is that it is possible to change $\hat{\tau}_{\text {MTD }}$ and $\hat{\tau}_{\text {MTFA }}$ by the design of the auxiliary input signal $\eta_{t}$. The mean values of $s_{t}$ and $c_{t}$ are directly proportional to the amplitude of $\eta_{t}$ when a parameter has changed in the system. In case $\hat{\tau}_{\text {MTD }}$ and $\hat{\tau}_{\text {MTFA }}$ are not satisfactory, it is possible to change them by changing the amplitude of $\eta_{t}$. The cost is an increase in the effect from $\eta_{t}$ on the external output $e_{t}$. This will not be possible in a passive FDI approach.

\section{Example}

The stochastic change detection method determined above will be illustrated using a simple example in the following. Consider a sampled version of a simple second-order system given by

$$
G(s)=\frac{k}{s^{2}+2 \zeta \psi s+\psi^{2}}=\frac{1}{s^{2}+0.2 s+1}
$$

influenced by stochastic disturbances. Variations in the three parameters $k, \zeta$ and $\psi$ will be considered.

In discrete time $\left(T_{s}=0.01 \mathrm{sec}\right)$ and in state space the system is given by

$$
\begin{aligned}
x_{t+1} & =y A x_{t}+B u_{t}+B d_{t}, \\
y_{t} & =C x_{t}+w_{t},
\end{aligned}
$$

where the noise processes are zero mean white noise sequences and

$$
\operatorname{Var}\left\{\left[\begin{array}{l}
d_{t} \\
w_{t}
\end{array}\right]\right\}=\left[\begin{array}{cc}
0.1 & 0 \\
0 & 0.01
\end{array}\right] .
$$

In this example the process noise is an input disturbance, but the methods are by no means restricted to this type. The control is based on a state estimate obtained by means of a stationary Kalman filter and the control is an ordinary LQ controller whose aim is to minimize the objective function

$$
J=\mathbf{E}\left\{\sum_{t=0}^{\infty} x_{t}^{T} Q x_{t}+u_{t}^{T} R u_{t}\right\}, \quad Q=I_{2}, \quad R=0.2 .
$$

This design results in a controller given by

$$
\begin{aligned}
& V=\frac{z^{2}-1.931 z+0.9332}{z^{2}-1.957 z+0.9581}, \\
& U=\frac{-0.2664 z+0.2661}{z^{2}-1.957 z+0.9581},
\end{aligned}
$$

and a model parameterized through

$$
\begin{aligned}
& N=\frac{5.05 z+5.046}{z^{2}-1.957 z+0.9581} 10^{-5} \\
& M=\frac{z^{2}-1.998 z+0.998}{z^{2}-1.957 z+0.9581} .
\end{aligned}
$$

A simple analysis of this closed-loop system results in residual variance equal to $\sigma_{0}^{2}=10^{-3}$ in the normal situation.

As mentioned in the previous section, the auxiliary signal was chosen to be a harmonic function, which has a distinct signature in the residual signal if a parameter change is present. The frequency was chosen by investigating the variation in $S(\omega, \theta)$ (see Figs. 46) in relation to $P_{e \eta}$ and $P_{\varepsilon d}$ over a range of frequencies and type of parameter changes. It was chosen to use the same harmonic function for both detection and isolation. It is therefore also relevant to consider the variation in $S(\omega, \theta)$ in the complex plane for different frequencies and parameter changes. Based on this analysis, the frequency was chosen to be $\omega_{0}=2.5 \mathrm{rad} / \mathrm{sec}$. The amplitude was chosen to be 0.64 , which is equivalent to having a power increase to a ten-fold level of the stochastic variance. The signals are plotted in Fig. 7 for the nominal case. It is clear that the residual $\left(\varepsilon_{t}\right)$ does not contain any signature of the auxilary signal.

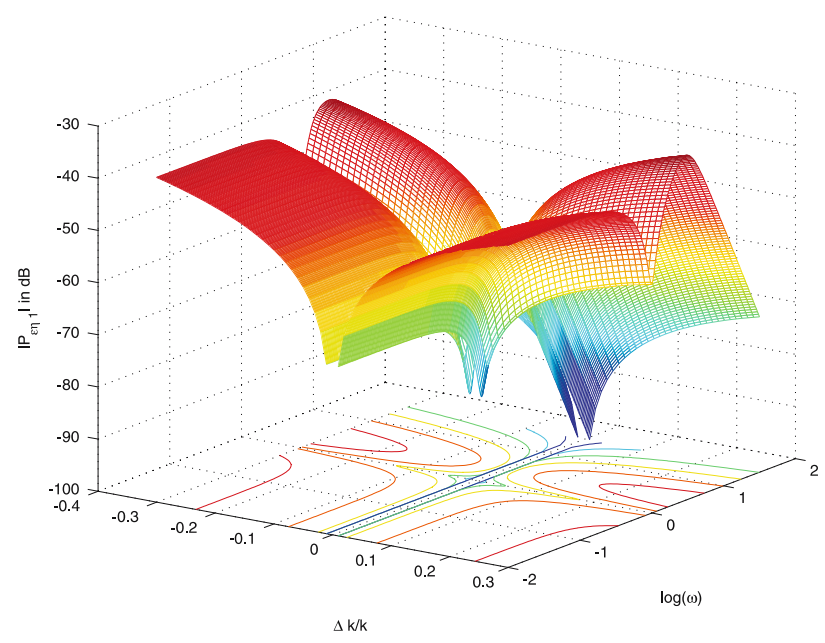

Fig. 4. Variation in $|S|$ as function of $\omega$ and $\Delta k / k$.

The parameters in the CUSUM detector were chosen to be

$$
\gamma=0.01, \quad B=50 .
$$

The choice of $\sigma_{1}$ was based on the knowledge of $\sigma_{0}^{2}$. The performance of the detector can be seen in Fig. 8, where the four detector signals (see (21) ) are well below the detection threshold. This is related to the fact that for these choices $\hat{\tau}_{\mathrm{MTFA}}=9181$. 


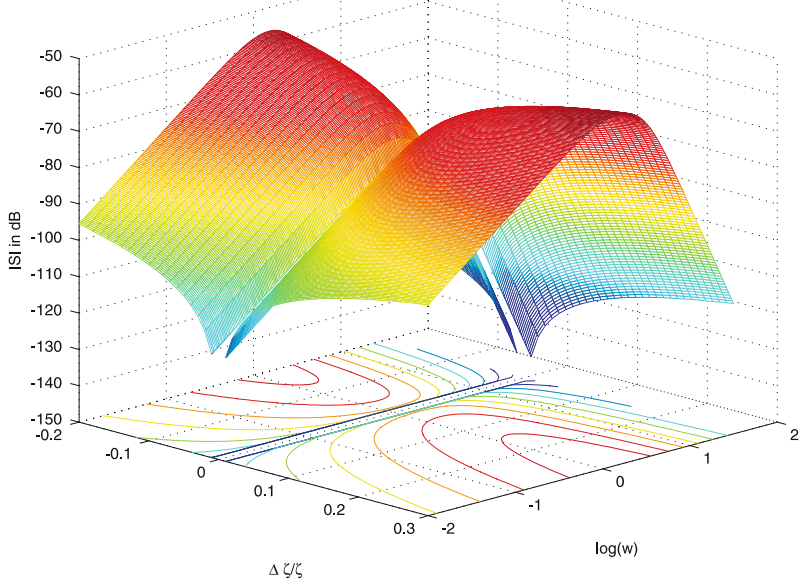

Fig. 5. Variation in $|S|$ as a function of $\omega$ and $\Delta \zeta / \zeta$.

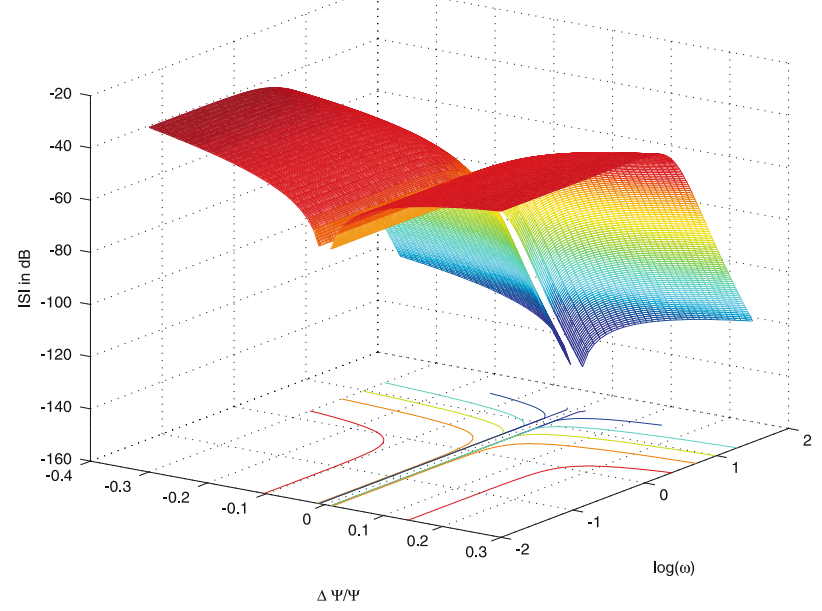

Fig. 6. Variation in $|\mathrm{S}|$ as a function of $\omega$ and $\Delta \Psi / \Psi$.
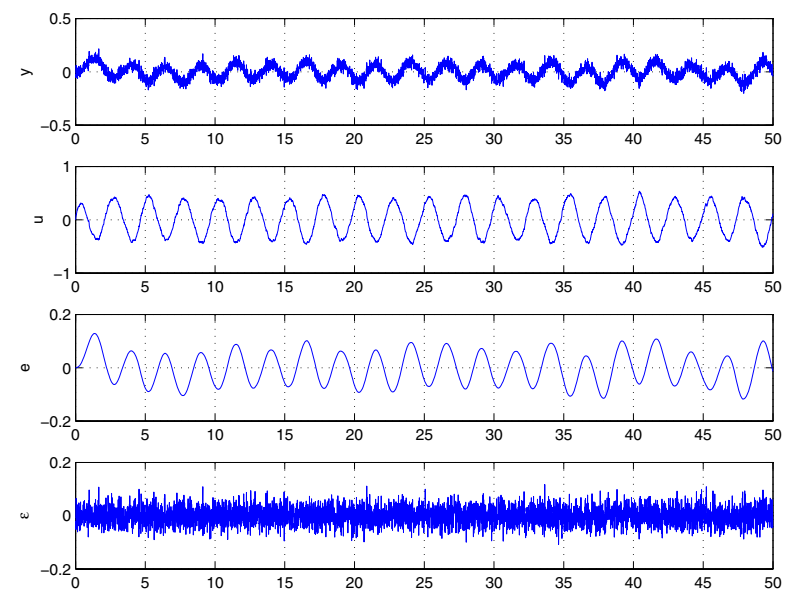

Fig. 7. Signals in the nominal situation.

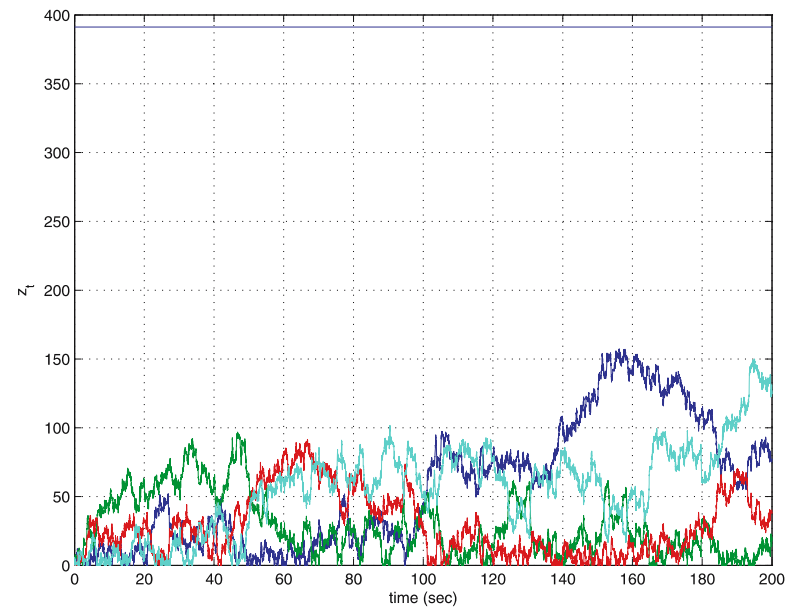

Fig. 8. CUSUM signals in a nominal situation.

Consider now an initial (at $t=0$ ) change in each of the parameters $k, \zeta$ and $\psi$. The detector signals are plotted in Figs. 911 for $10 \%, 50 \%$ and $10 \%$ changes in the respective parameters. Additionally, equivalent deterministic simulation results are given as well. The results of the three simulations are summarized in Table 1 Each row in this table is related to one type of parameter change (in $k, \zeta$ and $\psi$ ). The first column gives the channel number which signals a parameter change. The second column contains the time instant of detection, $t_{d}$, and the third column contains the estimate of $\tau_{M T D}$ in (25).

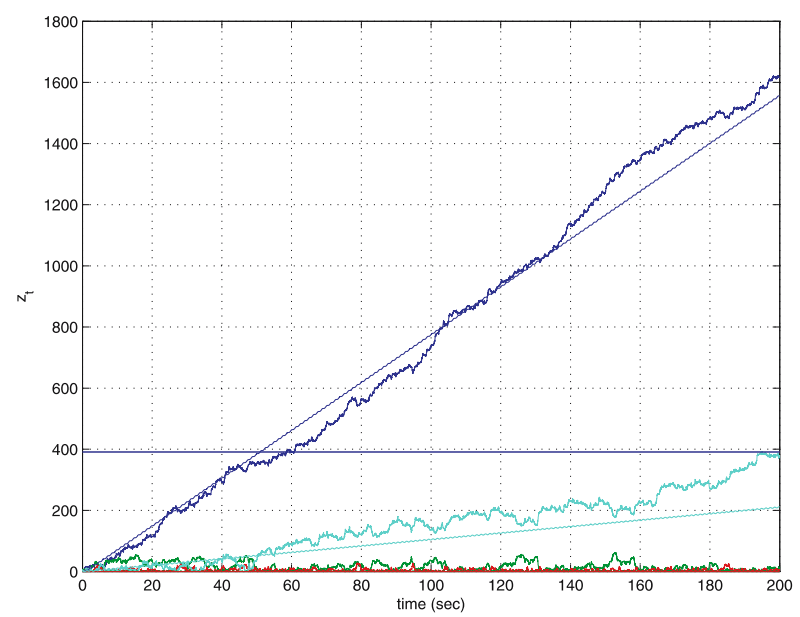

Fig. 9. CUSUM signals for a change in $k$.

In Fig. 12 $S(\omega, \theta)$ is shown in the complex plane for different parameter changes for $\omega_{0}=2.5 \mathrm{rad} / \mathrm{sec}$. As described in Section 4.2 the complex plane is divided into three double-coned areas with top at the origin. Each type of parameter change is assigned a designated unit vector (see Table 2). 


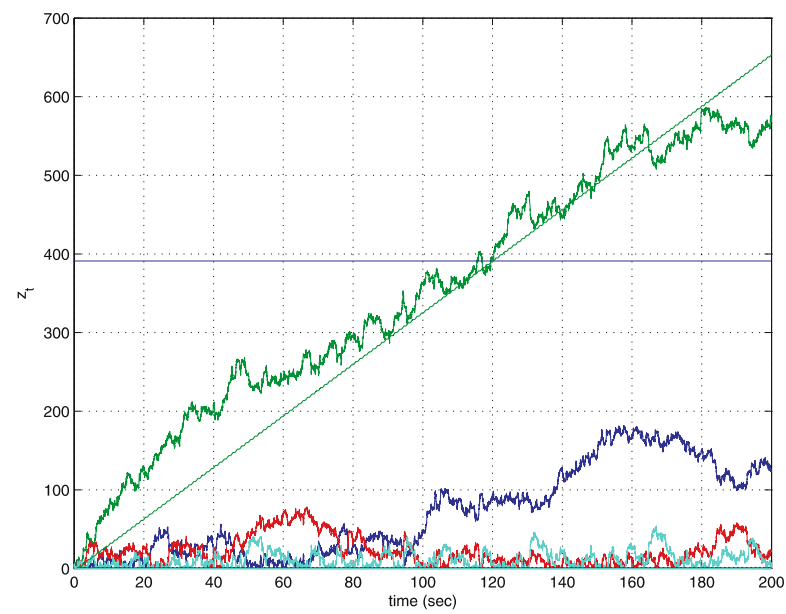

Fig. 10. CUSUM signals for a change in $\zeta$.

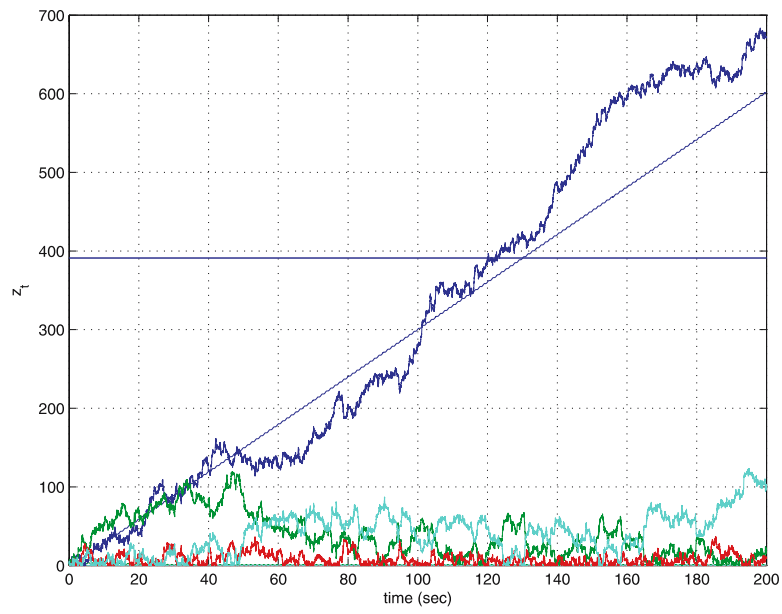

Fig. 11. CUSUM signals for a change in $\psi$.

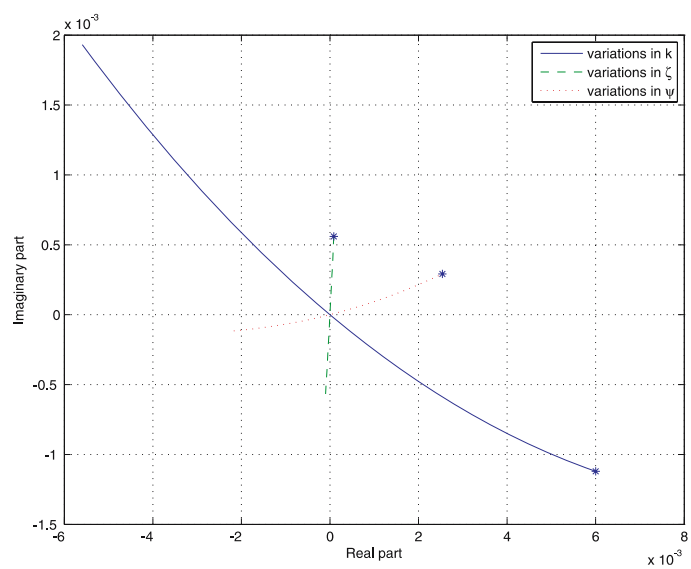

Fig. 12. Real and imaginary parts of $S$ for $\omega_{0}=2.5 \mathrm{rad} / \mathrm{sec}$ and three types of parameter changes (in $k, \zeta$ and $\psi$ ). The parameters vary from -0.1 to 0.1 on a relative scale. The $10 \%$ increase in the parameters is indicated with a star.
Table 1. Detection results.

\begin{tabular}{|c|c|c|c|}
\hline & Chanel & $t_{d}$ & $\hat{\tau}_{M T D}$ \\
\hline \hline Fig. & 1 & $58.60 \mathrm{sec}$ & $49.34 \mathrm{sec}$ \\
Fig.10 & 2 & $115.60 \mathrm{sec}$ & $115.01 \mathrm{sec}$ \\
Fig.11 & 1 & $120.12 \mathrm{sec}$ & $124.50 \mathrm{sec}$ \\
\hline
\end{tabular}

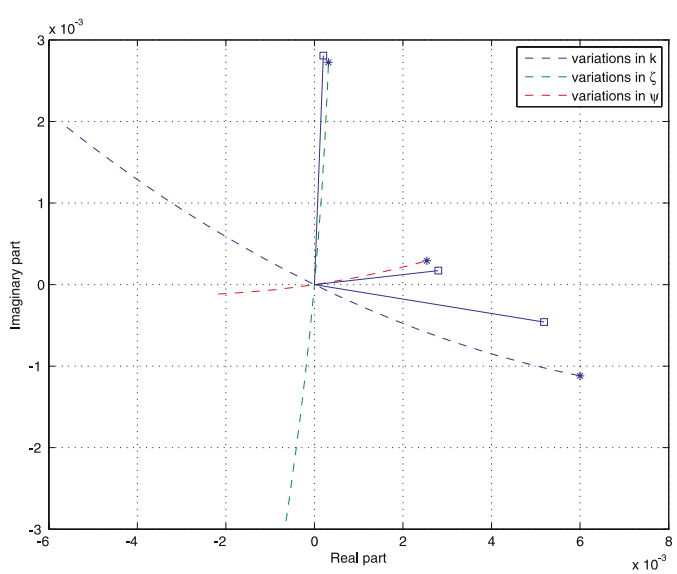

Fig. 13. Isolation signals for (individual) changes in $k, \zeta$ and $\psi$, respectively. The estimate is indicated with a box whereas the true signature is indicated with a star.

Table 2. Designated vectors $v_{i}, i=k, \zeta, \Psi$.

\begin{tabular}{|c|c|c|c|}
\hline & $k$ & $\zeta$ & $\psi$ \\
\hline \hline $\operatorname{Re}$ & 0.9830 & 0.1539 & 0.9934 \\
Imag & -0.1834 & 0.9881 & 0.1144 \\
\hline
\end{tabular}

When a parameter change has been detected and the parameter change instant, $T_{d}$, estimated, data from $\hat{T}_{d}$ to $t_{d}$ are used according to (22) to estimate the fault signature matrix, $S(\omega, \theta)$. The estimate of the time difference between the occurrence of the change, $\hat{T}_{d}$, and the detection hereof, $t_{d}$, is listed as the second column in Table 3 . This is illustrated in Fig. 13. The isolation is carried out as given by (23), which is a mechanization of finding the nearest fault curve. The results are summarized in Table 3, where each row corresponds to one type of parameter change (and one simulation). The columnwise data (the last 3 columns) are the projection of $v$ on each $v_{i}, i \in[1,2,3]$ in percent (with a sign). As could be predicted from Fig. [12, it is clear that it is harder to isolate changes in $k$ and $\psi$ than those in $\zeta$.

\section{Conclusion}

A new method for stochastic change detection and isolation in an AFD setting was described. The key issue is to use an auxiliary signal which has a distinct signature in 
Table 3. Isolation results, i.e., $v^{T} v_{i}$ in percent.

\begin{tabular}{|c|c|c|c|c|}
\hline & $t_{d}-\hat{T}_{d}$ & $k$ & $\zeta$ & $\psi$ \\
\hline \hline change in $k$ & $58.47 \mathrm{sec}$ & 48.77 & 3.24 & 47.99 \\
change in $\zeta$ & $115.55 \mathrm{sec}$ & -8.69 & 76.99 & 14.31 \\
change in $\psi$ & $115.59 \mathrm{sec}$ & 44.44 & 9.81 & 45.75 \\
\hline
\end{tabular}

the residual sequence, rather than a change in the variance or mean. The transfer function from an auxiliary input to a residual sequence equals the fault signature matrix which vanishes in the nominal case. The diagnosis is based on using both amplitude and phase information with respect to the signature in the residual output. Changes are detected and isolated by using a modified CUSUM test.

\section{References}

Basseville M. and Nikiforov I. (1993). Detection of Abrupt Changes: Theory and Application, Prentice Hall, Englewood Cliffs, NJ.

Campbell S., Horton K. and Nikoukhah R. (2002). Auxiliary signal design for rapid multi-model identification using optimization, Automatica 38(8): 1313-1325.

Campbell S., Horton K., Nikoukhah R. and Delebecque F. (2000). Rapid model selection and the separability index, Proceedings of the IFAC Symposium Safeprocess 2000, Budapest, Hungary, pp. 1187-1192.

Campbell S. and Nikoukhah R. (2004a). Auxiliary Signal Design for Failure Detection, Princeton University Press, Princeton, NJ.

Campbell S. and Nikoukhah R. (2004b). Software for auxiliary signal design, Proceedings of the American Control Conference, Boston, MA, USA, pp. 4414-4419.

Frank P. and Ding X. (1994). Frequency domain approach to optimally robust residual generation and evaluation for model-based fault diagnosis, Automatica 30(5): 789-804.

Gustafsson F. (2000). Adaptive Filtering and Change Detection, Wiley \& Sons, Chichester.
Kerestecioglu F. (1993). Change Detection and Input Design in Dynamic Systems, Research Studies Press, Baldock, Hertfordshire.

Kerestecioglu F. and Cetin I. (2004). Optimal input design for detection of changes towards unknown hypoteses, International Journal of System Science 35(7): 435-444.

Kerestecioglu F. and Zarrop M. (1994). Input design for detection of abrupt changes in dynamical systems, International Journal of Control 59(4): 1063-1084.

Niemann H. (2003). Dual Youla parameterization, IEE Proceedings - Control Theory and Applications 150(5): 493-497.

Niemann H. (2005). Fault tolerant control based on active fault diagnosis, Proceedings of the American Control Conference, Portland, OR, USA, pp. 2224-2229.

Niemann H. (2006). A setup for active fault diagnosis, IEEE Transactions on Automatic Control 51(9): 1572-1578.

Niemann H. and Poulsen N. (2005). Active fault diagnosis in closed-loop systems, Proceedings of the 16th IFAC World Congress, Prague, Czech Republic, (on DVD).

Nikoukhah R. (1994). Innovations generation in the presence of unknown inputs: Application to robust failure detection, Automatica 30(12): 1851-1867.

Nikoukhah R. (1998). Guaranteed active failure detection and isolation for linear dynamical systems, Automatica 34(11): 1345-1358.

Nikoukhah R., Campbell S. and Delebecque F. (2000). Detection signal design for failure detection: A robust approach, International Journal of Adaptive Control and Signal Processing 14: 701-724.

Poulsen N. and Niemann H. (2007). Stochastic change detection based on an active fault diagnosis approach, Proceedings of the 8th Conference on Diagnostics of Processes and Systems, DPS 2007, Słubice, Poland, pp. 113-120.

Tay T., Mareels I. and Moore J. (1997). High Performance Control, Birkhäuser, Boston, MA

Zhang X. (1989). Auxiliary Signal Design in Fault Detection and Diagnosis, Springer Verlag, Heidelberg.

Received: 10 October 2007 Revised: 29 January 2008 